\title{
Successful pre-emptive kidney transplantation in a cystinuria patient with nephrolithiasis-related end-stage renal disease
}

\author{
Masatoshi Matsunami ${ }^{1 *}$ (D), Kazuya Kinoshita ${ }^{1}$, Kiho Tanaka ${ }^{1}$, Yuki Nakamura ${ }^{1}$, Kenichi Ohashi ${ }^{3,5}$, Takeshi Fujii $^{3}$, \\ Yoshifumi Ubara ${ }^{2,4}$ and Yasuo Ishii ${ }^{1}$
}

\begin{abstract}
Background: Cystinuria is a rare autosomal recessive metabolic disorder that affects renal and intestinal cystine transport. Cystine stones are found in only $1-2 \%$ of all stone formers. Patients with cystinuria are at high risk for nephrolithiasis and subsequent morbidity. In spite of the various medical and surgical treatments that are currently available for cystinuria, some patients gradually develop kidney failure, with only a few reported cases regarding kidney transplantation (KTX) to treat end-stage renal disease (ESRD) secondary to cystinuria. Cystinuria is likely not to be a systemic disease; thus, renal replacement with transplantation seems a good therapeutic option for ESRD. However, few cystinuria patients have undergone KTx due to ESRD.

Case presentation: We herein describe the case of a 49-year-old man with cystinuria, frequent stone events, and ESRD who underwent pre-emptive ABO-incompatible kidney transplantation. At 2 years and 6 months post-transplantation, the patient remains asymptomatic with no prophylactic therapy for cystinuria, and the allograft function has been preserved without evidence of rejection.

Conclusions: In conclusion, a cystinuria patient with nephrolithiasis-related ESRD was successfully treated by transplantation. Although additional cases are required to confirm the efficacy of this approach, renal replacement may be useful for treating ESRD in patients with rare hereditary forms of kidney stone disease.
\end{abstract}

Keywords: Cystinuria, Nephrolithiasis, Kidney transplantation, Pre-emptive, ESRD

\section{Background}

Cystinuria is a rare, autosomal recessive hereditary disease that was first described by Archibald Garrod in 1908 [1]. It is also known as the most frequent monogenic cause of renal stones. The condition is present in approximately $1 \%$ of adults and $8 \%$ of children with nephrolithiasis. Globally, the average prevalence of cystinuria is approximately 1 per 7000 births; however, the prevalence ranges and widespread variation are observed [2,3]. This disease is caused by the impairment of the epithelial transport of dibasic amino acids (cystine, ornithine, arginine, and lysine)

\footnotetext{
* Correspondence: matsunami-m@toranomon.gr.jp

${ }^{1}$ Department of Surgery, Nephrology Center, Toranomon Hospital, 2-2-2

Toranomon, Minato-ku, Tokyo 105-8470, Japan

Full list of author information is available at the end of the article
}

in the renal proximal tubule and small intestine [2]. Because of its poor solubility at a typical urine $\mathrm{pH}$, cystine alone leads to urinary precipitation and recurrent nephrolithiasis that can cause obstruction, infection, and, ultimately, chronic kidney disease (CKD) [2, 4]. Usually, the diagnosis is based on the detection of urinary cystine crystals, a stone analysis, or increased urinary cystine levels. In some patients, stones develop into staghorn calculi [3].

Preventive medical managements include hydration (high fluid intake) and urinary alkalinization using orally administered potassium citrate. If hydration and urinary alkalinization fail to prevent cystine stone recurrence, the next step in the treatment should be the introduction of chelation or anti-urolithic therapy (such as tiopronin) to reduce the urinary concentration of cystine

(c) The Author(s). 2019 Open Access This article is distributed under the terms of the Creative Commons Attribution 4.0 International License (http://creativecommons.org/licenses/by/4.0/), which permits unrestricted use, distribution, and 
[3]. In cases of stone formation, large stones that are associated with pain, infection, or symptoms of obstruction require surgical intervention, such as extracorporeal shockwave lithotripsy (ESWL), percutaneous nephrolithotomy (PCNL), or endoscopic retrograde techniques [2]. In contrast, smaller asymptomatic stones may be monitored with close ultrasonographic follow-up [2].

End-stage renal disease (ESRD) seems to be an uncommon condition in cystinuria patients, and few studies have evaluated the comorbidities associated with cystinuria $[4,5]$. Our search of the PubMed database for English studies on successful kidney transplantation (KTx) for the treatment of ESRD secondary to cystinuria revealed only four previous reports [6-9]. The most recent case, that of a male aged in his 20s with bilateral staghorn calculi, was reported in 1993. Despite bilateral pyelolithotomy, the patient developed ESRD requiring renal replacement therapy (RRT) and eventually underwent KTx [8].

Thus, it is likely that cystinuria is not a systemic disease, making renal replacement with transplantation seems a good therapeutic option for ESRD due to cystinuria. We herein report a case in which an adult cystinuria patient with recurrent nephrolithiasis leading to ESRD was successfully treated by KTx.

\section{Case presentation}

The patient was a 49-year-old man from Japan with a lifelong and complicated history of cystinuria. He was diagnosed with cystinuria based on a stone analysis shortly after birth. His medical history was notable for multiple episodes of renal stones. He had no known family history of nephrolithiasis. At 1 year of age, he developed rightsided ureteric calculi and ureterolithotomy was performed. At 6 years of age, he suffered from left-sided ureteric calculi, and ureterolithotomy was performed as well. Despite the initiation of conservative treatment with potassium citrate and tiopronin, disease remission was not achieved. His next symptomatic stone event occurred at 23 years of age, when he was diagnosed with bilateral staghorn calculi. At this point, unfortunately, his rightsided kidney was already non-functioning. At 31 years of age, he had recurrent left-sided renal calculi and required PCNL for stone removal. At 39 years of age, the patient had an episode of right-sided renal abscess and was treated with intravenous antibiotic therapy with percutaneous nephrostomy (Fig. 1). His serum creatinine (Cr) gradually increased to approximately $1.8 \mathrm{mg} / \mathrm{dL}$. The patient was referred to our hospital for further investigation. Since there was a possibility of renal abscess recurrence, right-sided nephrectomy was eventually performed. The isolated kidney with obstructive pyelonephritis showed diffuse thinning of the renal parenchyma, interstitial fibrosis, hyalinization, and obscuring of the corticomedullary junction (Fig. 2). Thereafter, his renal function gradually declined and progressed to ESRD.

At 47 years of age, RRT was required because of frequent episodes of calculi, such as ureterolithiasis and nephrolithiasis, which ultimately resulted in ESRD. Both of the patient's parents carried a risk of having heterozygous mutations and were excluded as donors. However, his wife, a 45-year-old woman, volunteered to donate her kidney to him, so we decided to undergo preemptive living donor KTx.

The proposed transplant was $\mathrm{ABO}$ incompatible, from a donor with blood type A to a recipient with blood type B. At 2 weeks prior to transplantation, the administration of mycophenolate mofetil (MMF, $500 \mathrm{mg} /$ day) and the anti-CD20 monoclonal antibody rituximab (1 dose of $200 \mathrm{mg}$ ) was initiated according to our pretransplantation regimen (Fig. 3).

Preoperatively, tacrolimus (TAC) and methylprednisolone (mPSL) were also administered from 6 days before transplantation. TAC was started at a dose of $0.1 \mathrm{mg} / \mathrm{kg} /$ day and then increased to $0.15 \mathrm{mg} / \mathrm{kg} /$ day. Following 2 weeks of desensitization therapy, the patient underwent two sessions of hemodialysis (HD) and one session of double filtration plasmapheresis (DFPP) before transplantation.

In addition, induction therapy with intravenous methylprednisolone (mPSL [500 mg]) and basiliximab (1 dose of $20 \mathrm{mg}$ ) was administered on the day of transplantation.
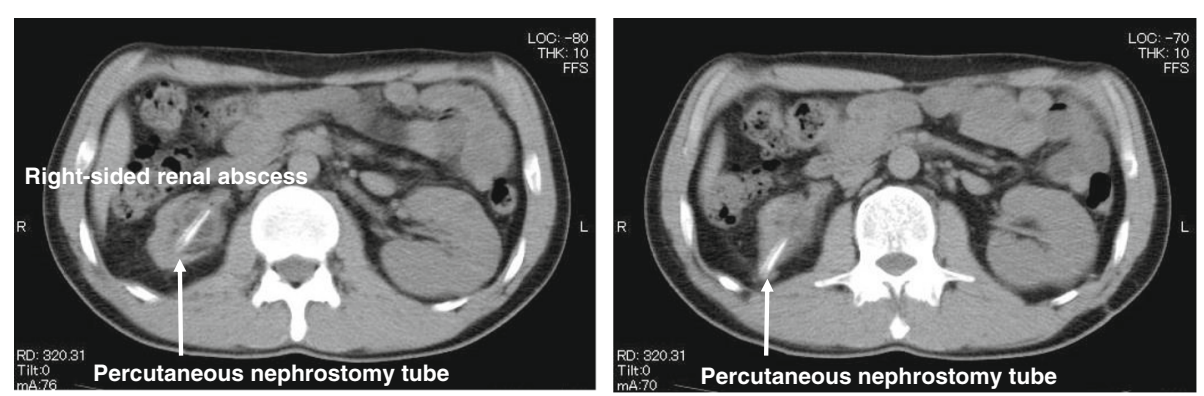

Fig. 1 CT scans of the right-sided renal abscess. A percutaneous nephrostomy tube was placed for drainage 

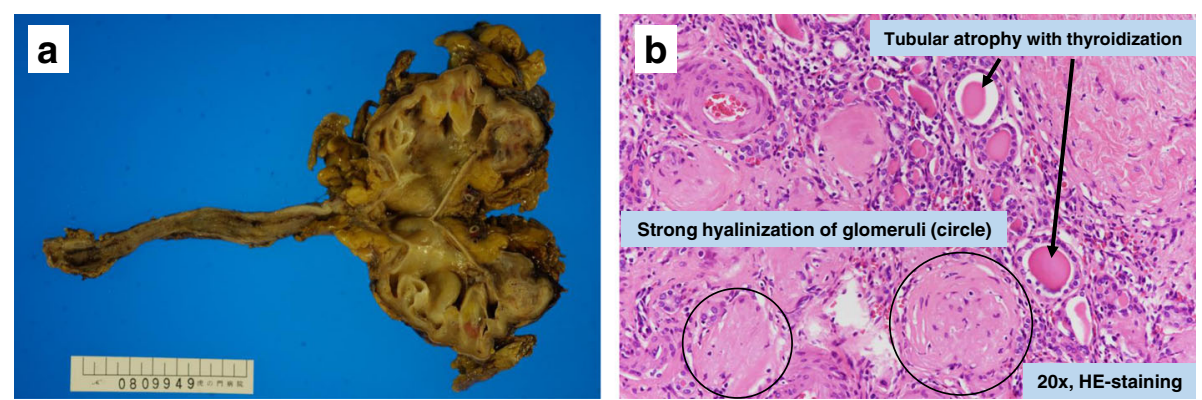

Fig. 2 The gross and microscopic observation of the isolated polycystic kidney. a The gross specimen showed a massively enlarged kidney with obstructive pyelonephritis. b Microscopic (histologic) observation showed wide interstitial fibrosis, tubular thyroidization, and tubular atrophy. Most glomeruli were hyalinized and collapsed

The renal graft was transplanted without incident into the left iliac fossa (because of a previous operation at the right iliac fossa). Subsequently, the graft became pink and the urine was immediately produced. The posttransplantation immunosuppression protocol consisted of TAC, MMF (1500 mg/day), and mPSL. Basiliximab was also administered 4 days after transplantation. The TAC trough level was maintained at $8-12 \mathrm{ng} / \mathrm{mL}$ for the first few weeks after transplantation. mPSL was gradually tapered to $5 \mathrm{mg}$ by the end of the post-transplantation period and switched to an oral formulation (Fig. 3).

Notably, the patient's $\mathrm{Cr}$ levels decreased after transplantation and are now maintained at $1.2 \mathrm{mg} / \mathrm{dL}$ under immunosuppression with a triple-drug regimen (TAC,
MMF, and mPSL) (Fig. 3). On post-operative days 180 and 544, a urinary amino acid analysis was performed and revealed that the patient's urinary amino acid levels remained within the normal ranges.

At present, 2 years and 6 months after KTx, the patient remains asymptomatic with no prophylactic therapy for cystinuria, and the allograft function has been preserved without evidence of rejection. On magnetic resonance angiography (MRA), there was no transplant renal artery stenosis, and the renal blood flow was maintained (Fig. 4). In addition, the hematologic follow-up has not demonstrated any significant adverse effects of immunosuppression (such as dyslipidemia or myelosuppression) or cytomegalovirus (CMV) infection events.

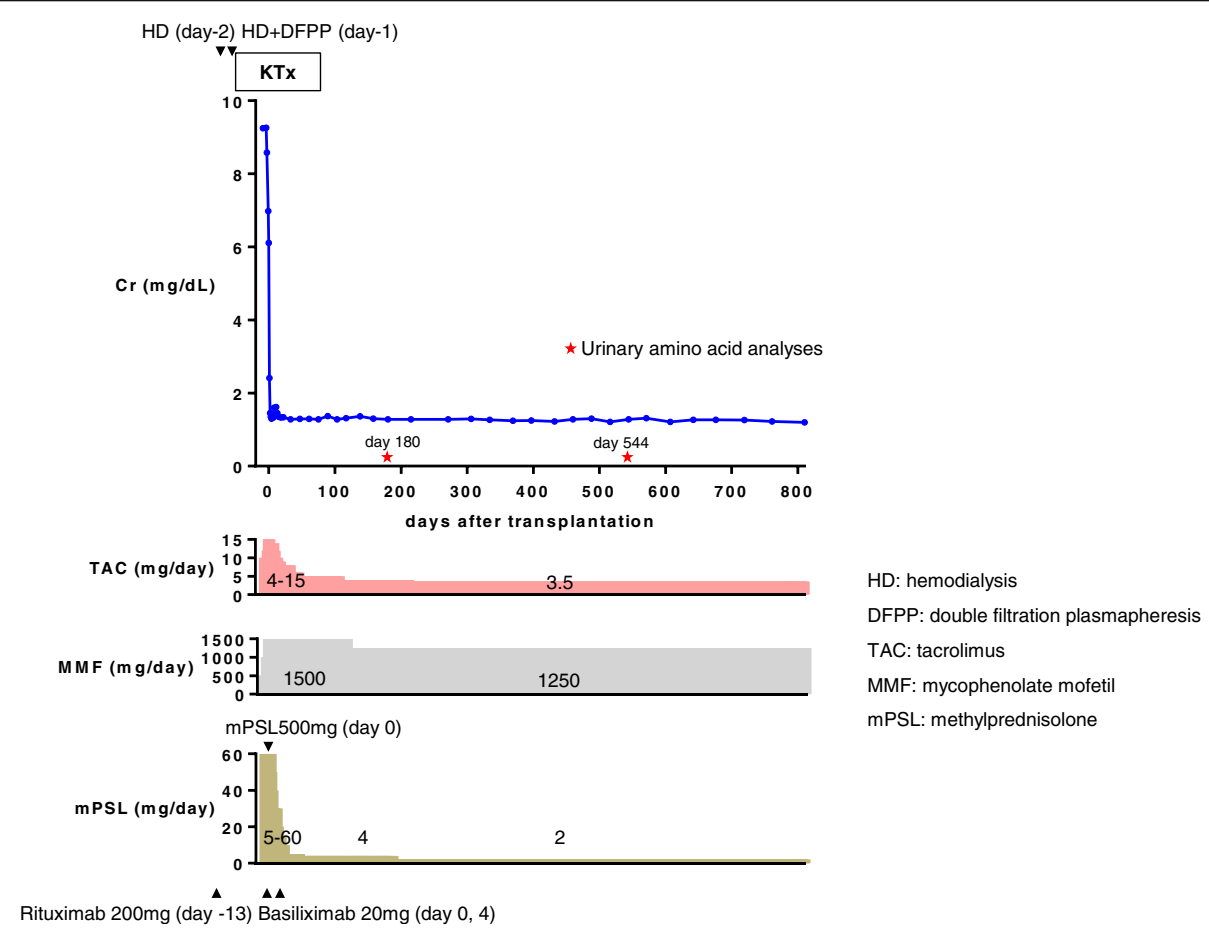

Fig. 3 Overview of the patient's clinical course and management. Tapering of triple immunosuppression (TAC, MMF, mPSL) with a stable serum creatinine level 


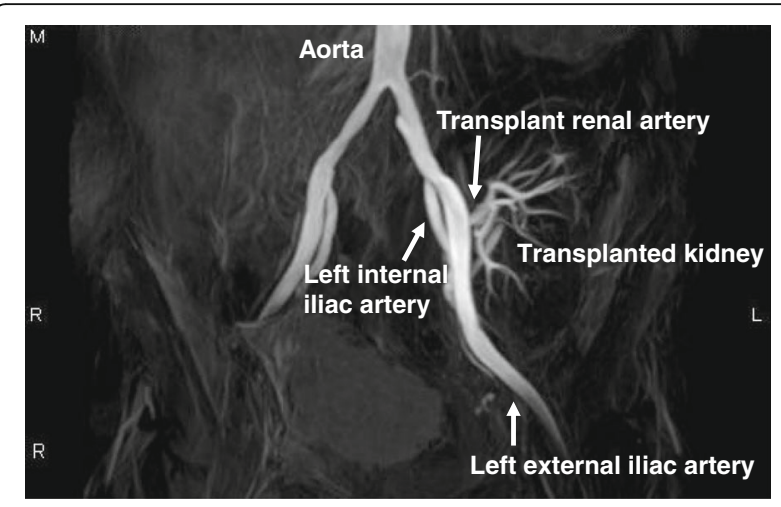

Fig. 4 MRA coronal image of a transplanted kidney. Renal artery anastomosis to the left external iliac artery showed no anastomotic stenosis

\section{Discussion and conclusions}

We presented the case of a cystinuria patient with nephrolithiasis-related ESRD who was followed for 2 years and 6 months after pre-emptive KTx. An accurate and early diagnosis of cystinuria is important for the long-term management of patients. In this case, the diagnosis of cystinuria was made based on a stone analysis and no genetic analysis was performed. The condition was characterized by lifelong, recurrent stone symptoms that were difficult to manage, both medically and surgically.

Patients with rare hereditary forms of kidney stone diseases, including primary hyperoxaluria, cystinuria, Dent disease, and adenine phosphoribosyltransferase deficiency, experience recurring stones-often starting in childhood-and are at high risk for CKD [5]. ESRD is common in primary hyperoxaluria, cystinuria, Dent disease, and adenine phosphoribosyltransferase deficiency. However, the CKD in cystinuria is usually less aggressive [5].

Recently, Prot-Bertoye et al. investigated CKD and its risk factors among a large series of French patients with cystinuria. This study showed that the prevalence of CKD in cystinuria patients was (26.7\%), but that only $1.1 \%$ (5 of 442) of cystinuria patients developed ESRD requiring RRT [4]. Among these CKD patients with cystinuria, the prevalence of hypertension (28.6\%) was high, and hypertension was found to be significantly associated with male sex, age, and CKD [4]. In this case, the cystinuria patient underwent nephrectomy, which has the potential to lead to ESRD. In addition to CKD, he also suffered from hypertension. Following transplantation, a blood pressure of $120 / 80-135 / 85 \mathrm{mmHg}$ has been maintained on Nifedipine (20 mg, once daily).

Kidney transplantation is the preferred treatment for ESRD. Our search of the literature only identified four cases in which KTx was performed for the treatment of ESRD secondary to cystinuria [6-9]. Among them, only one case resulted in graft failure 10 days after KTx due to acute rejection, not due to cystinuria, and the transplanted kidneys functioned well without recurrence of cystinuria for the remaining three cases (Table 1). These findings suggest that cystinuria does not develop in the transplanted kidneys with intact cystine transport.

In addition, as Tuso et al. described, hereditary kidney stone disease such as cystinuria is likely not to be a systemic disease, but causes kidney dysfunction [8]. Thus, we hypothesized that transplantation could be used to treat the patient's ESRD. Theoretically, there should be no recurrence of cystinuria, and in this case, the function of the allograft was nearly normal with normal urinary amino acid levels maintained for 2 years and 6 months after transplantation.

Meanwhile, since cystinuria is a hereditary disease, donors need to be carefully selected. In this case, we were not able to conduct genetic analysis because the patient's parents and wife did not consent. Despite the donor being the patient's wife, the patient is currently doing well with no subjective symptoms after transplantation.

According to recent studies, pre-emptive transplantation has many benefits, including improved patient and graft survival, and a less delayed graft function in comparison to transplantation after dialysis [10]. Other outcomes are improved, including a reduced overall cost of care, and improved patient employment status [11]. Despite the clearly defined benefits, the identification of a living donor is essential for successful pre-emptive transplantation. In Japan, patients can find the process of asking family members to donate a kidney to be daunting. Hence, the nephrologist or transplant surgeon should provide education on the donation process and the general safety of donation. If potential donors move through the process smoothly, then the likelihood of transplantation increases. In Japan, the rate of pre-emptive transplantation from

Table 1 Clinical characteristics of recipients with cystinuria after kidney transplantation

\begin{tabular}{|c|c|c|c|c|c|c|c|}
\hline Patient & Underlying disease & Age (years) & Sex & $\mathrm{KT} x$ & Cystinuria recurrence & Follow-up & Reference \\
\hline Case 1 & Cystinuria & 28 & $M$ & NA & No & 1 year and 8 months & Kelly et al. [6] \\
\hline Case 2 & Cystinuria & 38 & $M$ & DDKT & NA & Rejection (POD 10) & Hoitsma et al. [7] \\
\hline Case 3 & Cystinuria & $20 \mathrm{~s}$ & M & LDKT & No & 3 years and 6 months & Tuso et al. [8] \\
\hline Case 4 & Cystinuria & 46 & $F$ & DDKT & No & NA & Krizek et al. [9] \\
\hline
\end{tabular}

NA not available, DDKT deceased donor kidney transplantation, LDKT living donor kidney transplantation, POD post-operative days 
living donors has increased year by year and reached approximately $25.5 \%$ in 2013 [10, 12].

In conclusion, based on the excellent results that were observed, nephrolithiasis-related ESRD in a cystinuria patient was considered to have been successfully treated by transplantation. Although continued urinary amino acid analyses and long-term follow-up are needed to accurately determine the validity of KTx as a treatment for cystinuria, renal replacement may potentially be useful in patients who have rare hereditary forms of kidney stone diseases, including cystinuria. We hope that this case will be helpful to others facing the problem of deciding whether or not to attempt transplantation for the treatment of patients with a history of ESRD secondary to cystinuria. Furthermore, the accumulation of new reports in which good outcomes are achieved may help to establish KTx as an acceptable therapeutic option.

\section{Abbreviations}

CKD: Chronic kidney disease; ESRD: End-stage renal disease; KTx: Kidney transplantation; RRT: Renal replacement therapy

\section{Acknowledgements}

The authors would like to thank Dr. Atsushi Kato and Dr. Hideshi Ishizuka for their careful patient management at Saitama Shakaihoken Hospital (current JCHO Saitama Medical Center) in Saitama, Japan.

\section{Authors' contributions}

MM designed and wrote the manuscript. KK, KT, and YN treated the patient. $\mathrm{KO}$ and TF supplied the pathology reports. YU and YI supervised the transplant program and corrected the manuscript. All authors read and approved the final manuscript.

\section{Funding}

No funding was obtained for this study.

\section{Availability of data and materials}

All data supporting our findings are contained within the manuscript.

\section{Ethics approval and consent to participate}

Not applicable.

\section{Consent for publication}

Written informed consent was obtained from the patient to publish this case report and any accompanying images. A copy of the written consent form is available for review by the editor of this journal.

\section{Competing interests}

The authors declare that they have no competing interests.

\section{Author details}

'Department of Surgery, Nephrology Center, Toranomon Hospital, 2-2-2 Toranomon, Minato-ku, Tokyo 105-8470, Japan. ${ }^{2}$ Nephrology Center, Toranomon Hospital, Tokyo, Japan. ${ }^{3}$ Department of Pathology, Toranomon Hospital, Tokyo, Japan. ${ }^{4}$ Okinaka Memorial Institute for Medical Research, Tokyo, Japan. ${ }^{5}$ Department of Pathology, Yokohama City University Graduate School of Medicine, Yokohama, Japan.

Received: 11 March 2019 Accepted: 25 June 2019 Published online: 04 July 2019

\section{References}

1. Garrod A. The Croonian lectures on inborn errors of metabolism. Lancet. 1908;172(4427):1-7.
2. Chillaron J, Font-Llitjos M, Fort J, Zorzano A, Goldfarb DS, Nunes V, Palacin M. Pathophysiology and treatment of cystinuria. Nat Rev Nephrol. 2010;6(7):424-34.

3. Cochat P, Pichault V, Bacchetta J, Dubourg L, Sabot JF, Saban C, Daudon M, Liutkus A. Nephrolithiasis related to inborn metabolic diseases. Pediatr Nephrol. 2010;25(3):415-24.

4. Prot-Bertoye C, Lebbah S, Daudon M, Tostivint I, Bataille P, Bridoux F, Brignon P, Choquenet $C$, Cochat $\mathrm{P}$, Combe $\mathrm{C}$, et al. CKD and its risk factors among patients with cystinuria. Clin J Am Soc Nephrol. 2015;10(5):842-51.

5. Rule AD, Krambeck AE, Lieske JC. Chronic kidney disease in kidney stone formers. Clin J Am Soc Nephrol. 2011;6(8):2069-75.

6. Kelly S, Nolan EP. Excretory rates in posttransplant cystinuric patient. Jama. 1978;239(12):1132.

7. Hoitsma AJ, Koene RA, Trijbels FJ, Monnens LA. Disappearance of cystinuria after renal transplantation. Jama. 1983;250(5):615.

8. Tuso P, Barnett M, Yasunaga C, Nortman D. Cystinuria and renal transplantation. Nephron. 1993;63(4):478.

9. Krizek V, Erben J, Lazne M, Navratil P, Svab J. Disappearance of cystinuria after kidney transplantation. Br J Urol. 1983;55(5):575.

10. Goto N, Okada M, Yamamoto T, Tsujita M, Hiramitsu T, Narumi S, Katayama A, Kobayashi T, Uchida K, Watarai Y. Association of dialysis duration with outcomes after transplantation in a japanese cohort. Clin J Am Soc Nephrol. 2016;11(3):497-504.

11. Fishbane S, Nair V. Opportunities for increasing the rate of preemptive kidney transplantation. Clin J Am Soc Nephrol. 2018;13(8):1280-2.

12. Yagisawa T, Mieno M, Yoshimura N, Yuzawa K, Takahara S. Current status of kidney transplantation in Japan in 2015: the data of the Kidney Transplant Registry Committee, Japanese Society for Clinical Renal Transplantation and the Japan Society for Transplantation. Ren Replace Ther. 2016;2(1):68.

\section{Publisher's Note}

Springer Nature remains neutral with regard to jurisdictional claims in published maps and institutional affiliations.

Ready to submit your research? Choose BMC and benefit from:

- fast, convenient online submission

- thorough peer review by experienced researchers in your field

- rapid publication on acceptance

- support for research data, including large and complex data types

- gold Open Access which fosters wider collaboration and increased citations

- maximum visibility for your research: over $100 \mathrm{M}$ website views per year

At BMC, research is always in progress.

Learn more biomedcentral.com/submissions 